\title{
A generalized dose-response relationship for adenovirus infection and illness by exposure pathway
}

\author{
P. TEUNIS ${ }^{1,2 *}$, J. SCHIJVEN ${ }^{2,3}$ AND S. RUTJES ${ }^{2}$ \\ ${ }^{1}$ Centre for Infectious Disease Control, National Institute of Public Health and the Environment (RIVM), \\ Bilthoven, The Netherlands \\ ${ }^{2}$ Center of Global Safe WASH, Hubert Department of Global Health, Rollins School of Public Health, Emory \\ University, Atlanta, GA, USA \\ ${ }^{3}$ Environmental Hydrogeology Group, Department of Earth Sciences, Faculty of Geosciences, Utrecht University, \\ Utrecht, The Netherlands
}

Received 14 March 2016; Final revision 17 June 2016; Accepted 27 July 2016;

first published online 30 August 2016

\section{SUMMARY}

Adenoviruses are found everywhere in the environment, and cause various health problems including symptoms of enteric illness, and respiratory illness. Despite their significance to public health, few studies have addressed the health risks associated with exposure to adenovirus. Human challenge studies have been published for a few adenoviruses, which involved exposure through oral ingestion, inhalation, intranasal and intraocular droplet inoculation. Nothwithstanding the different symptoms resulting from such exposures, infection can be defined as colonization of a corresponding mucosa. A two-level doseresponse model was developed to describe the distributions of infectivity and pathogenicity in various challenge studies of adenovirus, incorporating differences in inoculation route as shift in average infectivity and pathogenicity. This dose-response model can be used to make predictions for the infectivity of adenovirus, specific to any of the four studied inoculation methods. The generalized adenovirus dose-response relationship for infection and acute illness takes into account variation in infectivity and/or pathogenicity across adenovirus types, as well as uncertainty due to limited data.

Key words: Adenoviruses, risk assessment, statistics, virus infection.

\section{INTRODUCTION}

Several types of adenovirus have been demonstrated to infect humans [1-3] and cause a wide variety of common and sporadic infections. Adenoviruses can infect various sites of the respiratory tract [4], the gastrointestinal tract [5-7], and the eye [8]. They can also infect the urinary bladder and the liver, although less frequently. Currently, there are 57 identified human adenovirus serotypes, which can be divided into seven subgenera (A-G) [9]. It is not known why certain serotypes cause

\footnotetext{
* Author for correspondence: Dr P. F. M. Teunis, RIVM, PO Box 1, 3720BA Bilthoven, The Netherlands.

(Email: peter.teunis@rivm.nl)
}

disease in some organs and not in others. The mechanisms of such tissue tropism or organ-specific pathogenicity cannot be explained by tissue culture experiments, animal models or bioinformatics analysis.

There is quite some information on the abundant presence of adenoviruses in the environment. It is well recognized that adenoviruses are very stable in watery suspension due to their well-organized capsid structure without envelope. They have been shown to remain infectious in several water types, such as surface, recreational and drinking water for a prolonged period of time, and have been shown to cause numerous waterborne outbreaks. It is therefore interesting to consider the health risks associated with their presence in the environment $[10,11]$. 
If any adenovirus is found in an environmental water sample, and its concentration can be quantified $[12,13]$, one may wish to translate human exposure into health risk. A complicating factor is found in the fact that adenovirus can infect humans via various exposure pathways. To predict the risk of infection, or the risk of acute symptoms given a certain (mode of) exposure, a dose-response relationship is needed. Aside from the magnitude of the dose, the probabilities of infection and illness may depend on the exposed mucosal tissues (the mode of exposure) and on the virus type, as well as on host factors determining susceptibility for that particular exposure mode.

Data on clinical consequences of human exposure to adenoviruses are uncommon, and for many pathogens there is limited information, usually from experiments with volunteers, done during the second half of the 20 th century $[14,15]$. From the literature, only five different adenovirus challenge studies are available. These studies used one virus type belonging to subgenus $\mathrm{E}$ (AdV4) and two different adenoviruses belonging to subgenus B (AdV7 and 16) that were inoculated via four different exposure modes: oral ingestion (Oral), inhalation (Inhal), intranasal (Nasal) and intraocular droplet inoculation (Ocul). For any of these virus type/exposure type combinations, a dose-response relationship could be described. Due to the diversity in conditions, any such dose-response model by virus/exposure type would be based on limited data and thus be highly uncertain.

The objective of this study was to develop a generalized dose-response model from these studies, to provide a basis for translating exposure to adenovirus into risk of infection and/or acute illness. By treating the set of dose-response studies as a (small) sample from the universe of adenovirus dose-response relationships. we may generalize the dose-response relationship from this collection of observed data. Using a hierarchical Bayesian framework, we will show that such generalization is possible and that, despite the small numbers of studies, we find meaningful differences in the effects of different exposure routes.

\section{Adenovirus challenge studies data}

The combined studies included a total of 91 subjects challenged in 23 dose groups. The studies are listed in Table 1 with information on dose, numbers exposed, numbers infected and numbers of subjects with acute illness symptoms.
Table 1. Adenovirus challenge studies with references. Three different virus types inoculated by four different pathways. Data include: estimated (mean) dose, numbers challenged (Exp), numbers shedding virus (Inf) and numbers of subjects with acute symptoms (IIl)

\begin{tabular}{cccllll}
\hline \hline Ref. & Virus & Inoculation & Dose & Exp & Inf & Ill \\
\hline [17] & AdV4 & Oral & $10^{5.8}-10^{7.2}$ & 3 & 3 & 0 \\
& AdV7 & Oral & $10^{5.8}-10^{6.2}$ & 15 & 15 & 0 \\
[20] & AdV4 & Nasal & 2500 & 2 & 2 & 0 \\
& & & 250 & 3 & 0 & 0 \\
& AdV7 & Nasal & 150000 & 2 & 2 & 0 \\
& & & 1500 & 2 & 2 & 0 \\
& & & 150 & 2 & 1 & 0 \\
& & & 15 & 2 & 0 & 0 \\
& & & 15000 & 3 & 3 & 0 \\
[18] & AdV4 & Inhal & 150 & 2 & 1 & 0 \\
& & & 5 & 3 & 3 & 3 \\
& & & 1 & 3 & 3 & 3 \\
& & & 11 & 1 & 1 \\
[16] & AdV16 & Ocular & 150000 & 3 & 3 & 3 \\
& AdV4 & Nasal & 400 & 3 & 3 & 0 \\
& & & 79 & 3 & 2 & 1 \\
& & & 14 & 2 & 1 & 0 \\
& & & 10 & 2 & 0 & 0 \\
& & & 3 & 6 & 0 & 1 \\
& & Inhal & 171 & 4 & 4 & 4 \\
& & & $5-11$ & 9 & 9 & 8 \\
& & & $1-2$ & 5 & 3 & 3 \\
\hline \hline & & & 0.1 & 3 & 0 & 0 \\
\hline
\end{tabular}

Titration of the inocula was similar in all papers: studies [16-19] expressed dose in $\mathrm{TCID}_{50}$ units in human embryonic kidney cells, while study [20] reported $\mathrm{TCID}_{50}$ in pig kidney cells (PK).

Oral ingestion failed to produce any clinical symptoms [17], neither fever nor diarrhoea (one subject was reported to have had a brief diarrheal episode which was attributed to a different cause). Aerosol inoculation (Inhal) produced respiratory symptoms in the upper or lower respiratory tract (similar to pneumonia), sometimes accompanied by fever [18, 19]. Intranasal inoculation did not produce any severe symptoms, some subjects had common cold-like symptoms that were attributed to other causes [20]. All subjects inoculated intra-ocularly developed conjuctivitis [16].

It is worthwhile noting the considerable range in doses, and the differences in dosing schedules in the different experiments. It should also be noted that the data of oral and intra-ocular inoculation provide very limited information of the dose-response relationship. 


\section{METHODS}

\section{Dose-response models for infectious pathogens}

Risk assessment rests on the analysis of conditional events: illness is conditional on (i.e. can only happen in) an infected host. Similarly, infection is conditional on exposure. Many human enteric pathogens infect their hosts at low doses: exposure to few organisms is associated with a considerable risk of infection $[21,22]$. As a consequence, the probability of exposure to at least a single infectious particle limits the probability of any health effect (infection, acute illness) in microbial dose-response assessment. At an average dose estimated from the concentration of suspended pathogens $(c)$ multiplied by the inoculated volume $(V)$, the probability of not ingesting even a single pathogen can be calculated. For instance, for a wellmixed (Poisson) suspension

$P_{\exp }(c V)=1-\mathrm{e}^{-c V}$,

At low doses this probability of exposure imposes a 'natural' limit to the risk of infection, independent of the infectivity of the pathogen or the susceptibility of the host [23].

If any ingested pathogen has a fixed probability $p_{m}$ of surviving the chain of barriers to infection (numbered $p_{1}, \ldots, p_{\mathrm{m}}$ ) the dose-response relationship for infection is

$P_{\text {inf }}\left(c V \mid p_{m}\right)=1-\mathrm{e}^{-p_{m} c V}$,

where the parameter $p_{m}$ quantifies host-pathogen interaction. When there is heterogeneity in this interaction (there usually is), a mixing distribution can be used, leading to the beta Poisson model for microbial infection

$P_{\text {inf }}(c V \mid \alpha, \beta)=1-{ }_{1} F_{1}(\alpha, \alpha+\beta ;-c V)$.

The dose-response relationship for the probability of acute symptoms in infected individuals (the conditional probability of illness, given infection) is defined as

$P_{\text {ill } \mid \text { inf }}(c V \mid r, \eta)=1-\left(1+\frac{c V}{\eta}\right)^{-r}$,

based on the hazard of becoming ill, proportional to the duration of infection [24].

\section{Development of a generalized dose-response model}

Within a single dose-response experiment the number of infected subjects is a binomial sample out of those exposed, with dose-dependent probability of infection $P_{\text {inf }}(c V \mid \alpha, \beta)$. Likewise, the number of symptomatic subjects is a binomial sample out of those infected, with dose-dependent probability $P_{\text {inf } \mid \text { ill }}(c V \mid r, \eta)$. The likelihood thus is the product of the two binomial probabilities, over all dose groups in the experiment.

To let each separate dose-response experiment (by inoculation method and virus type) contribute to the generalized dose-response relationship, the data are analysed jointly in a hierarchical framework [15, 25]. Each study is thus assigned their own dose-response relationship, for infection as well as for illness. Variation in the infection parameters $(\alpha, \beta)$ and illness parameters $(r, \eta)$ in experiments is modelled by assuming they have a joint distribution. This joint distribution may be interpreted as the 'group'-level distribution of infectivity and pathogenicity, with the data as a sample from the 'universe' of adenovirus dose-response relationships [25].

To improve estimation, parameters were transformed as in [26]:

$$
\begin{array}{ll}
u_{1}=\alpha /(\alpha+\beta) & u_{2}=r /(r+\eta), \\
v_{1}=\alpha+\beta & v_{2}=r+\eta, \\
w_{1}=\log \left[u_{1} /\left(1-u_{1}\right)\right] & w_{2}=\log \left[u_{2} /\left(1-u_{2}\right)\right], \\
z_{1}=\log \left(v_{1}\right) & z_{2}=\log \left(v_{2}\right),
\end{array}
$$

so that we are estimating the mean value $\left(u_{1}\right)$ of the beta distribution for $p_{m}$ and a quantity that is inversely related to its variance (for large positive $v_{1}$ the variance tends to zero). Further $u_{1}$ was logit-transformed and $v_{1} \log$-transformed. The illness parameters $(\eta, r)$ were given the same transformation.

The effect of inoculation route was incorporated by assuming that the mean of the transformed parameter $w_{1}$, $w_{2}$ is categorical, where $\mu_{w_{1}}$,inoc and $\mu_{w_{2}}$,inoc can each have four different values, corresponding to the inoculation method (oral ingestion, intranasal droplet inoculation, aerosol inhalation, or intraocular droplet inoculation). As a simplifying assumption, the variation parameters $z_{1}, z_{2}$ were assumed to be the same for all eight experiments (as the small numbers of doses tested were not expected to allow assessment of the variation in infectivity and pathogenicity for each separate experiment).

The hierarchical model was implemented in a Bayesian framework, using normal priors for $w_{1}, w_{2}$ and $z_{1}, z_{2}$ with fixed s.D. $=2$. Uncorrelated normal $($ mean $=0$, s.D. $=4)$ hyperpriors were taken for $\mu_{w_{1}}$, $\mu_{w_{2}}$. Posterior parameter samples were obtained using JAGS [27], controlled through rjags [28] in R [29]. More details can be found in the last section of the Appendix. 
Table 2. Parameter estimates (mean and 95\% range) for the adenovirus dose-response, by inoculation route: infectivity (infection dose-response) parameters $(\alpha, \beta)$ and pathogenicity (conditional illness dose-response) parameters $(\eta, r)$

\begin{tabular}{lll}
\hline \hline Inoculation & $\alpha$ & $\beta$ \\
\hline Oral & $5.11(0.006-39.6)$ & $2.80(0.00002-14.1)$ \\
Nasal & $1.27(0.0002-8.44)$ & $5.27(0.01-37.9)$ \\
Inhal & $5.24(0.004-28.2)$ & $2.95(0.001-25.8)$ \\
Ocul & $5.43(0.002-40.0)$ & $1.88(0.00003-14.3)$ \\
Inoculation & $\eta$ & $r$ \\
\hline Oral & $6.53(0.01-49.2)$ & $0.41(0.000001-2.36)$ \\
Nasal & $6.40(0.006-41.5)$ & $1.48(0.00002-8.08)$ \\
Inhal & $3.36(0.0008-24.7)$ & $3.04(0.0007-25.7)$ \\
Ocul & $2.40(0.000006-16.5)$ & $3.42(0.001-27.9)$ \\
\hline \hline
\end{tabular}

\section{RESULTS}

Parameter estimates are listed in Table 2, for the infectivity and conditional illness parameters by exposure category: oral ingestion, nasal droplet inoculation, aerosol inhalation, and ocular droplet inoculation. Estimates for the separate studies can be found in Appendix Table A1.

Corresponding dose-response relationships are shown in Figures 1 and 2. Figure 1 shows the doseresponse relationship for infection by exposure route. Although the $95 \%$ (posterior predictive) ranges appear similar, for intranasal droplet inoculation the estimated median probability of infection is lower as for the other three exposure pathways.

The probability of illness as a function of dose can be calculated by multiplying the infection probability [eqn (3)] and the conditional probability of illness given infection [eqn (4)]. Figure 2( $a-d)$ shows these unconditional illness dose-response relationships for the four inoculation routes.

Here too the $95 \%$ (posterior predictive) intervals are similar, but the median curves indicate that oral and intranasal droplet exposure are less pathogenic than inhalation or ocular droplet exposure.

This pattern is confirmed by calculating the doses required to cause a $1 \%$ and a $50 \%$ risk, of infection and of illness. These predicted InfD01 and InfD50, and IllD01 and IllD50 are given in Figure 3. Corresponding numbers are tabulated in Table 3. The doses required for infection are low: few infectious virus particles; intranasal droplets appear less infectious. For acute symptoms, higher doses are necessary, with oral and intranasal droplets showing the lowest pathogenicity (i.e. the highest IllD01 and IllD50).

\section{DISCUSSION}

Data from five studies on three different adenovirus types and four different modes of inoculation were analysed jointly with separate dose-response models for infection and for illness (conditional on infection). The model was organized into four categories, representing the four different modes of inoculation: oral ingestion, intranasal droplet inoculation, inhalation of aerosolized virus, and intraocular droplet inoculation. Note that these modes of inoculation lead to different illness endpoints: enteric symptoms (oral exposure), upper respiratory tract symptoms (intrasal droplets, aerosol inhalation), and conjunctivitis (intraoclar droplets). To overcome the lack of information on the shape of the dose-response relationship in each separate experiment for adenovirus infection and illness, we modelled the effect of inoculation mode as a shift in location of the infection and illness dose-response relationships, assuming that their shapes are common in all virus types and inoculation routes. In this manner the generalized dose-response model based on the hierarchical framework utilizes information across studies, weighting information of each study not only by the numbers of subjects or numbers of dose groups, but by the amount of information they provide for the joint distribution of the hyperparameters defining the 'group'-level dose-response relationship. This allows incorporation of studies contributing information that cannot be combined by pooling of data, because of heterogeneity in the data subsets [30].

Obviously, the amount of information provided by the observed data is variable: in the studies on oral ingestion and ocular droplet inoculation all exposed individuals were infected, resulting in limited information on dose-response, whereas the nasal droplets and aerosol inhalation studies are much richer in 
(a)

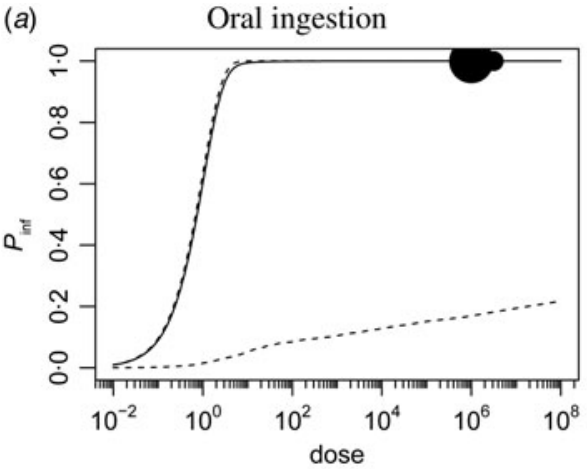

(c)

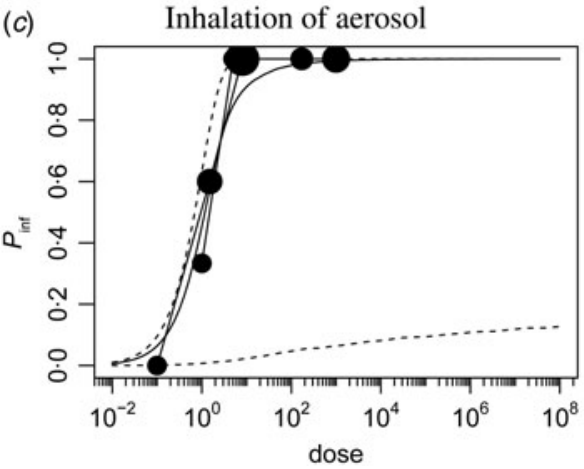

(b) Nasal droplet inoculation

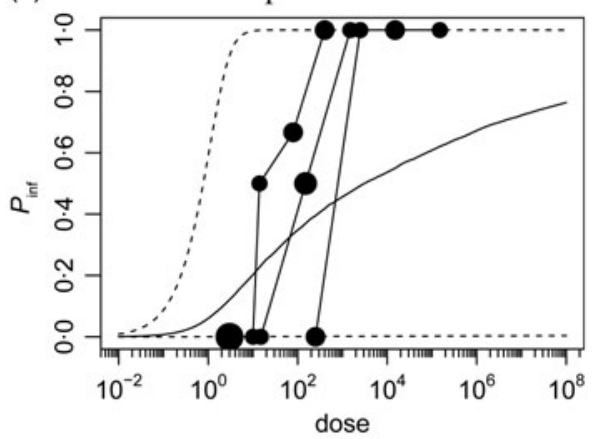

(d) Ocular droplet inoculation

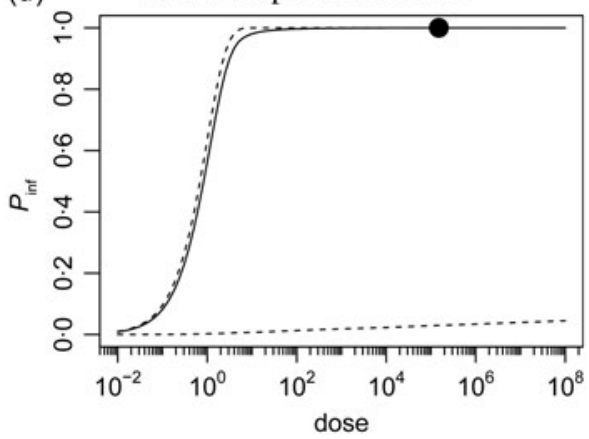

Fig. 1. Dose-response relationship for infection via the four different inoculation routes. Each graph shows the median and $95 \%$ range of the probability of infection as a function of dose, and any available data, as a bubble chart (symbol size proportional to the numbers challenged). Observations from the same study are connected.
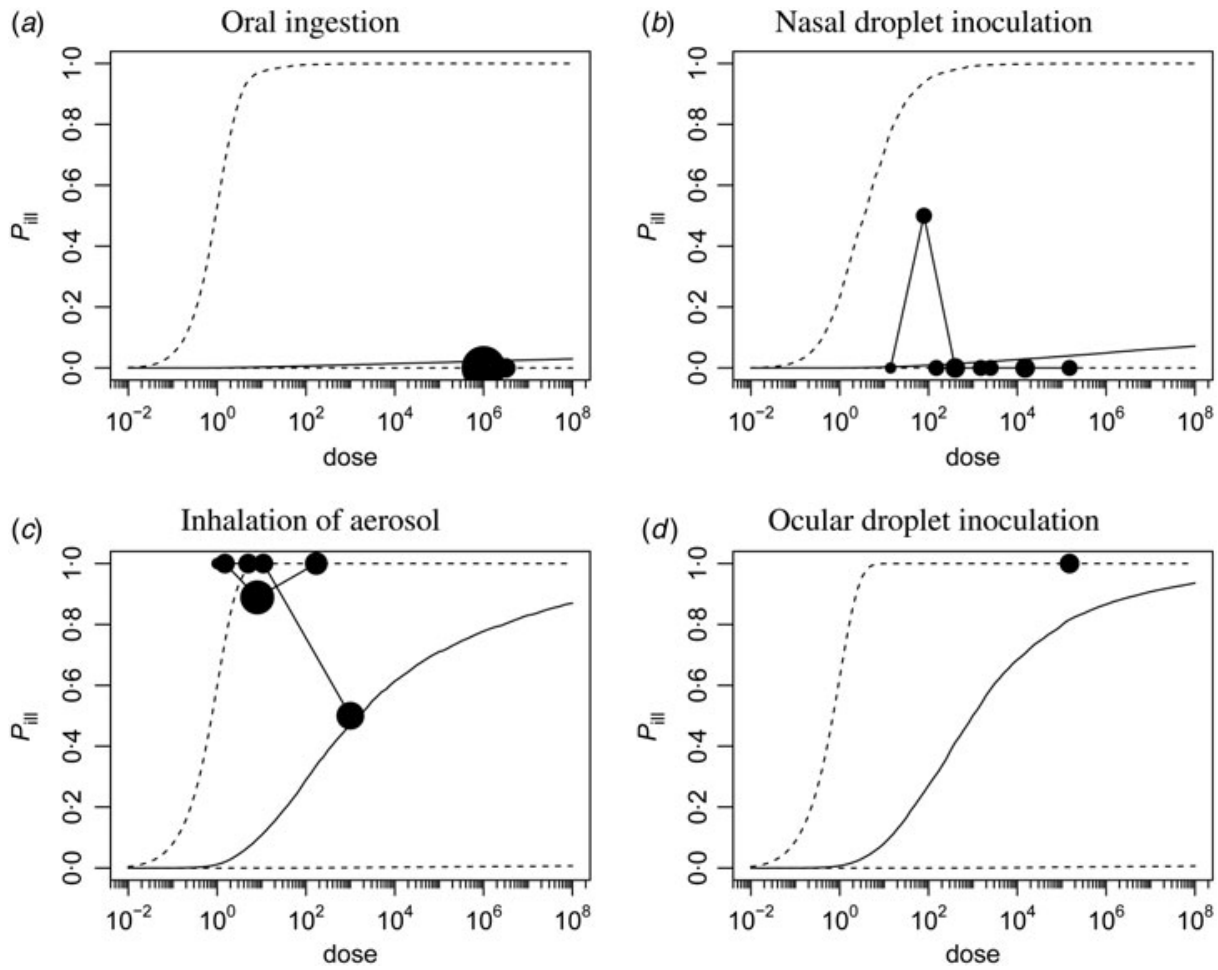

Fig. 2. Dose-response relationship for illness via the four different inoculation routes. Each graph shows the median and $95 \%$ range of the (unconditional) probability of illness as a function of dose, and any available data, as a bubble chart (symbol size proportional to the numbers challenged). Observations from the same study are connected. 
(a)

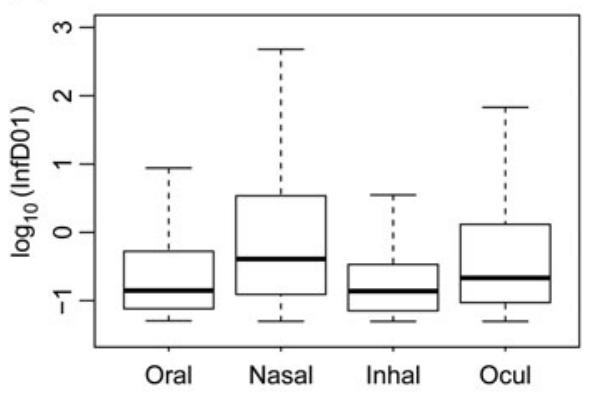

(c)

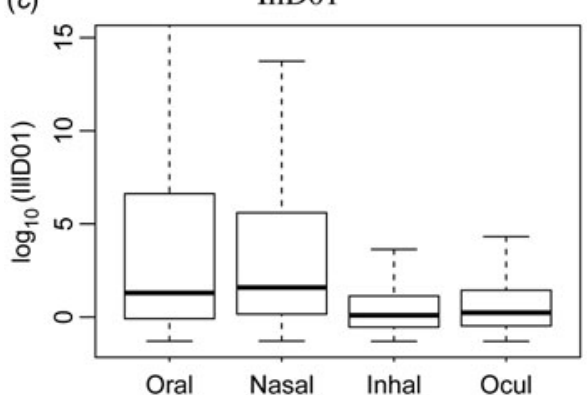

(b)

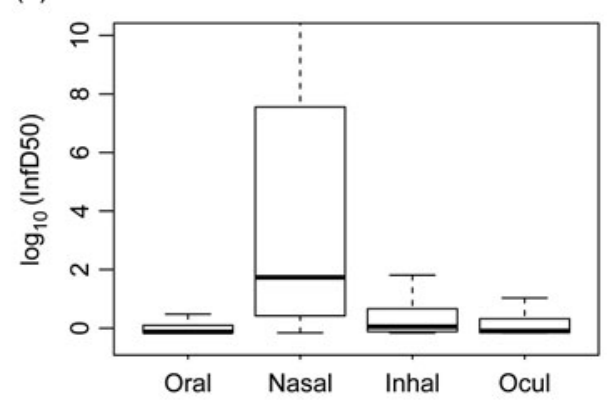

(d)

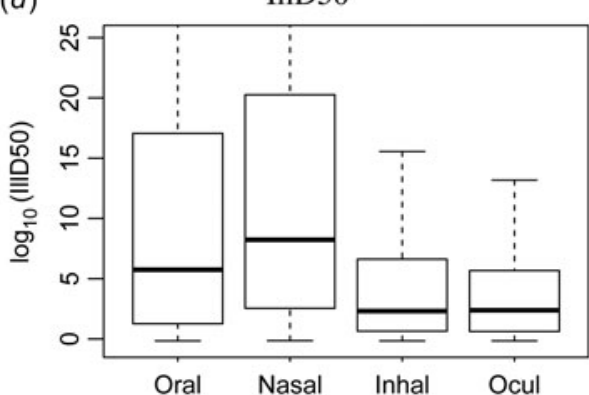

Fig. 3. Box plots of estimated doses for $1 \%$ and $50 \%$ probability of infection $(a, b)$ and illness $(c, d)$ via the four different exposure routes. Shown are median (horizontal line), quartiles (box) and $95 \%$ range (whiskers).

Table 3. Estimated dose required to cause 1\% and 50\% infection risk (InfD01 and InfD 50) and 1\% and 50\% illness risk (IllD01 and IllD50) for adenovirus, by inoculation route: mean and 90\% range

\begin{tabular}{lll}
\hline \hline Inoculation & InfD01 & InfD50 \\
\hline Oral & $0.14(0.055-814.0)$ & $0.74\left(0.69-1.05 \times 10^{4}\right)$ \\
Nasal & $0.41\left(0.059-1.22 \times 10^{8}\right)$ & $54.3\left(0.84-8.81 \times 10^{28}\right)$ \\
Inhal & $0.14(0.053-20.8)$ & $1.12\left(0.70-1.13 \times 10^{10}\right)$ \\
Ocul & $0.21\left(0.059-3.90 \times 10^{4}\right)$ & $0.80\left(0.69-5.61 \times 10^{9}\right)$ \\
Inoculation & IllD01 & IllD50 \\
\hline Oral & $20.0\left(0.12-3.81 \times 10^{27}\right)$ & $5.62 \times 10^{5}\left(0.84-3.49 \times 10^{37}\right)$ \\
Nasal & $38.7\left(0.17-2.06 \times 10^{24}\right)$ & $1.73 \times 10^{8}\left(2.96-1.15 \times 10^{37}\right)$ \\
Inhal & $1.25\left(0.074-1.11 \times 10^{5}\right)$ & $210.0\left(0.84-1.80 \times 10^{26}\right)$ \\
Ocul & $1.73\left(0.084-2.25 \times 10^{4}\right)$ & $239.4\left(0.75-3.64 \times 10^{25}\right)$ \\
\hline \hline
\end{tabular}

information. However, it should be noted that first, illness is conditional on infection: a symptomatic response can only occur in an infected subject. Therefore illness outcomes provide indirect information on infection. Second, the hierarchical framework allows sharing information among studies: by assuming that inoculation route affects only the mean values of the location parameters for infection $(w)$ and illness $(\log (\eta))$ we have implied that the remaining parameters are independent of inoculation route, and may be estimated from all observations jointly. Both the oral and ocular droplet inoculation data do not show trend [31]: they only provide information that the applied doses are sufficient for causing $100 \%$ infection. Doses of the same magnitude also suffice to cause infection in all exposed via the other inoculation pathways, so that exclusion of the oral and ocular droplet inoculation data would have little effect on the remaining dose-response relationships. However, absence of illness after oral challenge is informative of low pathogenicity, at the high doses used in this study.

Comparing predicted infectivities, for instance by comparing InfD50s (Fig. 3a) shows that inoculation 
via oral ingestion is highly efficient: a low dose may lead to a high probability of infection. The same is true for inhalation of aerosolized virus, and intraocular droplet inoculation. Intranasal droplets seem to require somewhat higher doses (50 times higher, on average) to achieve the same $(50 \%)$ probability of infection.

The dose required to cause $50 \%$ illness (acute symptoms, of a kind corresponding to the mode of inoculation) is highest for intranasal droplet inoculation, and lowest for inhalation of aerosolized virus. The ratio of mean IllD50s is $\sim 10^{6}$. Note that there is considerable uncertainty associated with each of these estimates.

When data are available on environmental concentrations of adenovirus, and there is an exposure model predicting doses for a given exposure route, the models presented here may allow translation of these doses into risk of infection and acute illness. What if the environmental adenovirus is a different type, perhaps related to any of the studied ones, but not the same? Because variation associated with virus type is incorporated into the hierarchical dose-response model, using the generalized categories (oral, nasal, inhalation, ocular) includes such virus type effects. It has to be noted, however, that such generalization is based on a small sample (of only three different virus strains).
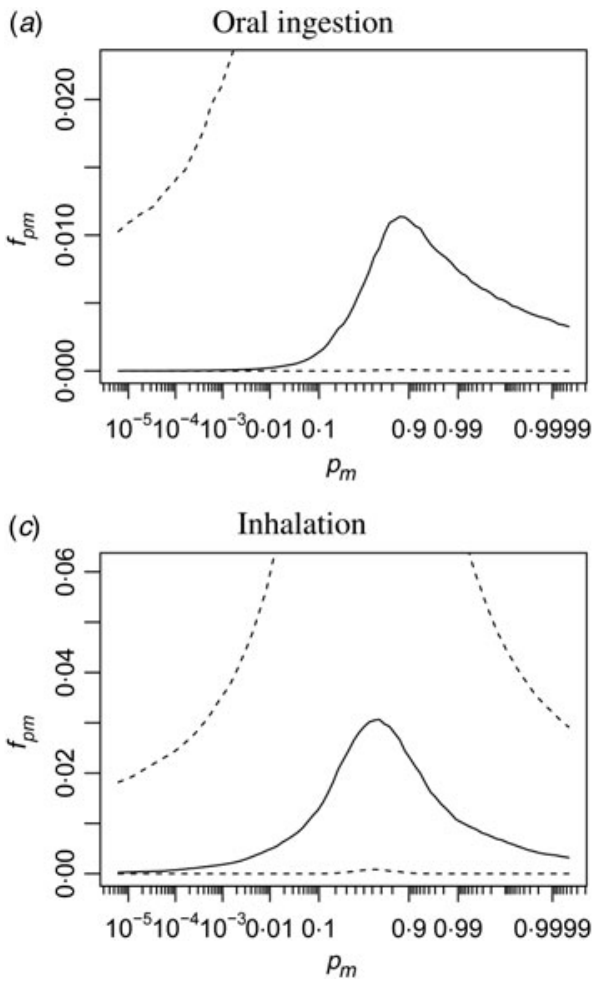

It would be interesting to see how infectivity and subsequent illness of AdV40 and 41, two enteric adenoviruses belonging to subgenus $\mathrm{F}$, would fit into these generalized dose-response relationships of the four different pathways, especially since these enteric variants may be most relevant for environmental transmission by exposure to different water sources.

In conclusion, adenovirus appears highly infectious, when delivered via oral ingestion, but at the same time the probability that such low-dose infection results in acute enteric symptoms is low in the four modes of inoculation studied. Among respiratory endpoints, inhalation of aerosolized virus appears more efficient than intranasal droplets, both in achieving infection and in subsequently causing symptoms of respiratory illness.

\section{APPENDIX}

\section{Infectivity estimates $\left(p_{m}\right)$}

In Figure A1 infectivity is illustrated in a different manner: here the (beta) distribution of the single-hit infectivity $\left[p_{m}\right.$ in eqn (2)] is shown, for each of the four inoculation routes.

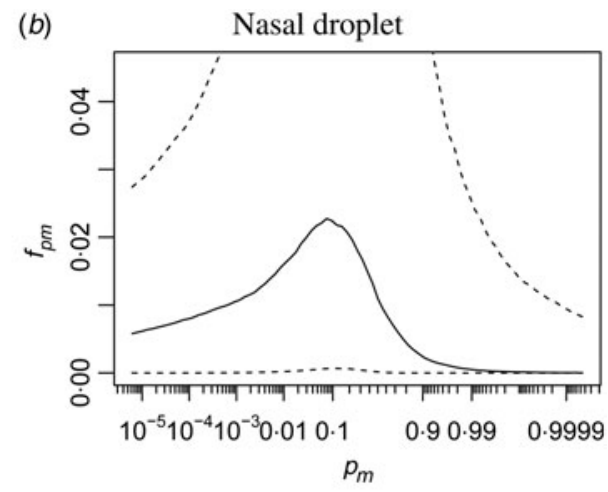

(d)

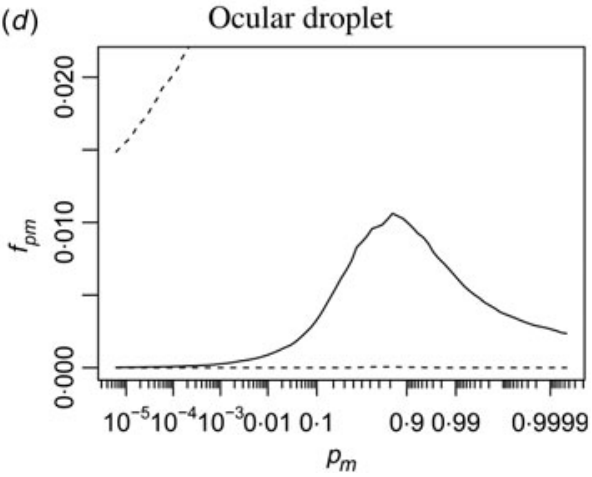

Fig. A1. Distributions of infectivities via the four different inoculation routes. Quantiles of the beta density (median, and $95 \%$ range) of the single-hit probability $p_{m}$ are shown, on a logit scale. 


\section{Parameter estimates for separate experiments}

Table A1. Parameter estimates (mean and 95\% range) for the separate adenovirus dose-response studies: infectivity (infection dose-response) parameters $(\alpha, \beta)$ and pathogenicity (conditional illness dose-response) parameters $(\eta, r$ )

\begin{tabular}{llll}
\hline \hline Virus & Inoculation & $\alpha$ & $\beta$ \\
\hline AdV4 & Oral & $6.63(0.02-42.2)$ & $2.36(0.00002-17.2)$ \\
AdV7 & Oral & $6.81(0.03-51.0)$ & $3.11(0.00002-25.4)$ \\
AdV4 & Nasal & $0.09(0.004-0.36)$ & $7.17(0.02-50.6)$ \\
AdV7 & Nasal & $0.47(0.07-1.83)$ & $29.7(0.13-216.1)$ \\
AdV4 & Inhal & $2.78(0.008-22.8)$ & $2.30(0.002-19.5)$ \\
AdV16 & Ocul & $5.12(0.04-36.8)$ & $5.01(0.00003-34.3)$ \\
AdV4 & Nasal & $0.65(0.04-2.63)$ & $21.2(0.15-89.8)$ \\
AdV7 & Inhal & $6.27(0.13-44.5)$ & $5.37(0.02-39.3)$ \\
Virus & Inoculation & $\eta$ & $r$ \\
\hline AdV4 & & $0.007(0.0000008-0.036)$ \\
AdV7 & Oral & $2.47(0.006-18.7)$ & $0.002(0.000001-0.09)$ \\
AdV4 & Oral & $1.68(0.006-12.3)$ & $0.46(0.00001-1.72)$ \\
AdV7 & Nasal & $3.34(0.002-21.9)$ & $0.007(0.000008-0.047)$ \\
AdV4 & Nasal & $1.14(0.0003-8.40)$ & $7.06(0.13-46.6)$ \\
AdV16 & Inhal & $9.51(0.076-57.8)$ & $8.43(0.097-47.5)$ \\
AdV4 & Ocul & $5.12(0.0002-38.5)$ & $1.00(0.008-8.21)$ \\
AdV7 & Nasal & $12.7(0.077-82.9)$ & $8.79(0.40-60.0)$ \\
\hline \hline
\end{tabular}

\section{Separate experiments (Figs A2-A4)}

Figures A2-A4 show infection dose-response, illness dose-response, and doses for $1 \%$ and $50 \%$ probability of infection and illness, respectively.

\section{Source code for the JAGS model}

Posterior parameter samples were obtained using JAGS v. 4.2 .0 [27], controlled through rjags v. 4-5 [28] in R v. 3.2.3 [29]. After a burn-in of $10^{4}$ iterations, three chains were run in parallel, each with $10^{6}$ iterations, and thinning $10^{3}$, producing 3000 posterior samples of all parameters. Convergence was checked by Gelman and Geweke diagnostics (using the CODA package). Aside from the data (Table A1) that comprised eight experiments, four additional experiments were added, each consisting of only missing data, for each of the four exposure pathways. The parameters estimated for these four added experiments could be used for prediction, as these were sampled from the 'group' distributions for the four exposure pathways (because these consisted of missing data, not contributing to the likelihood in a Bayesian model). 

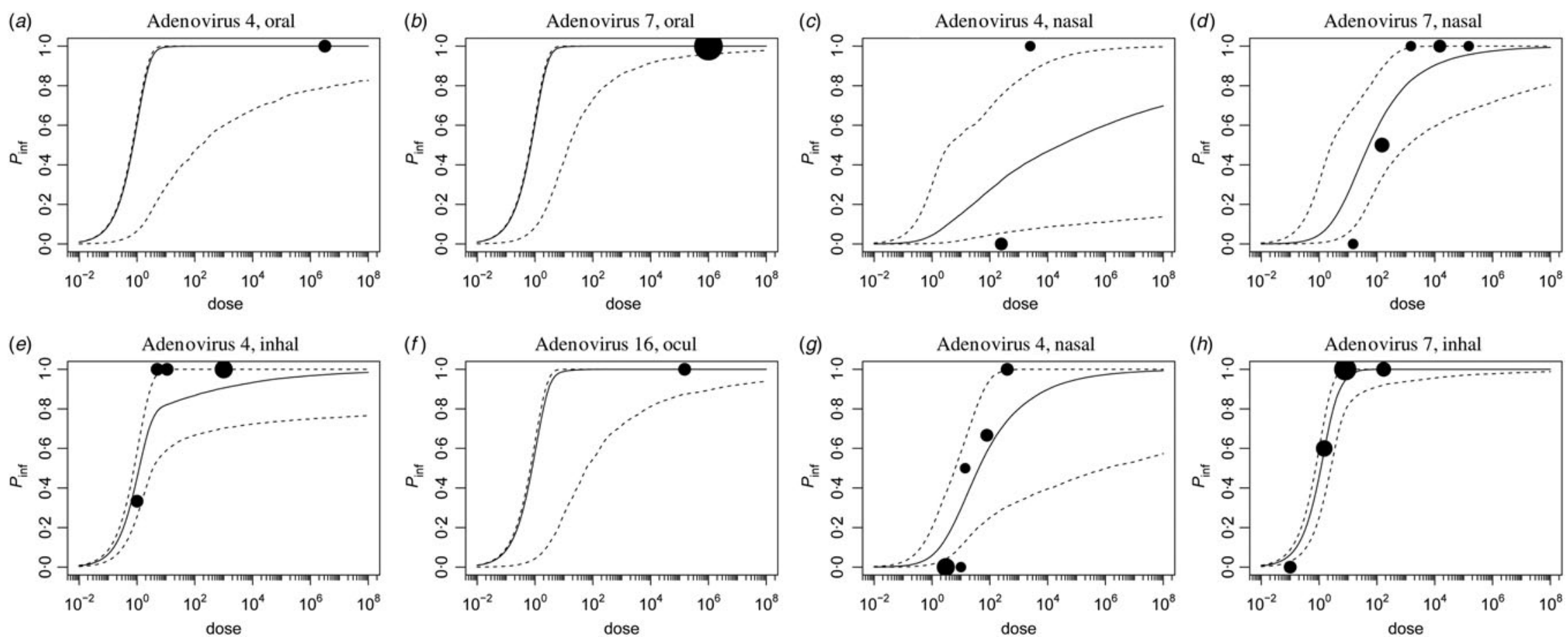

Fig. A2. Infection dose-response, separate studies. 
(a)
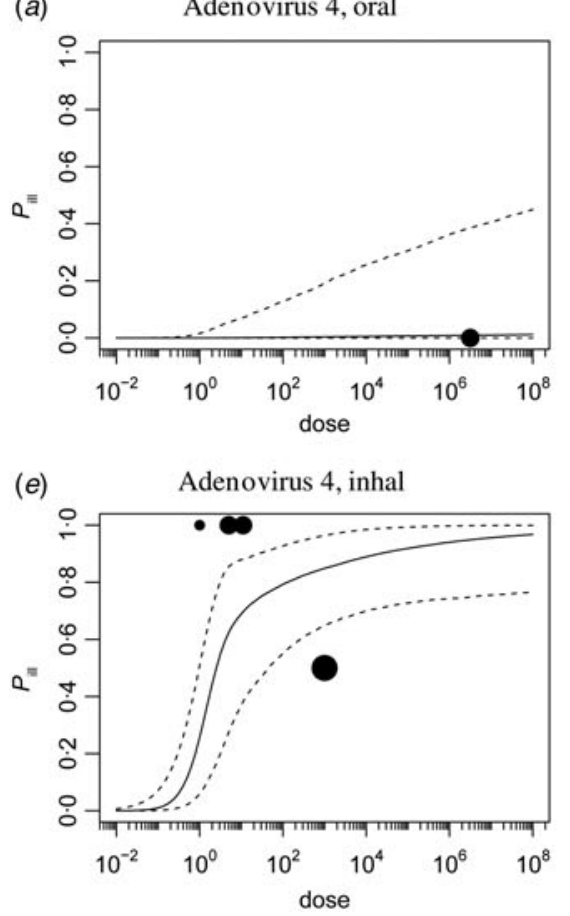

(b)
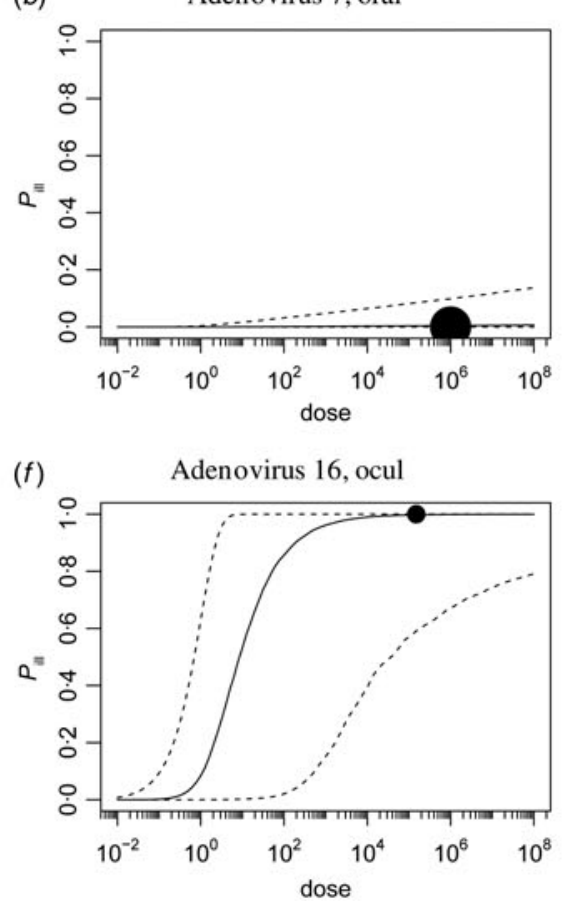

(c)

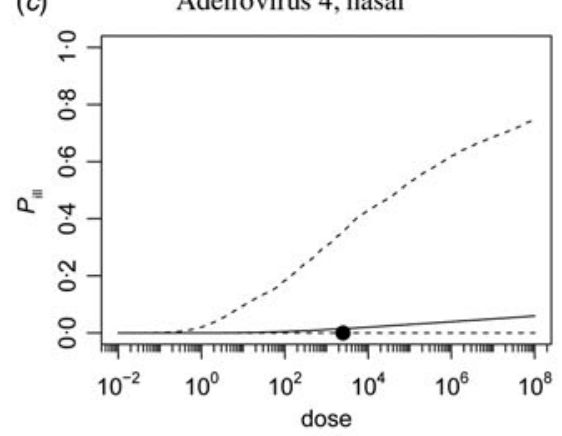

(g)

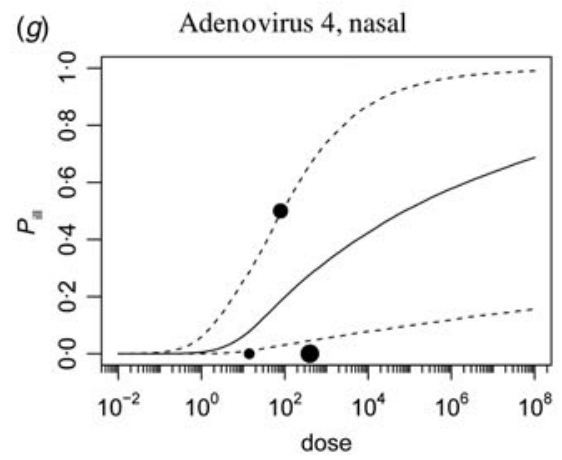

(d) Adenovirus 7, nasal
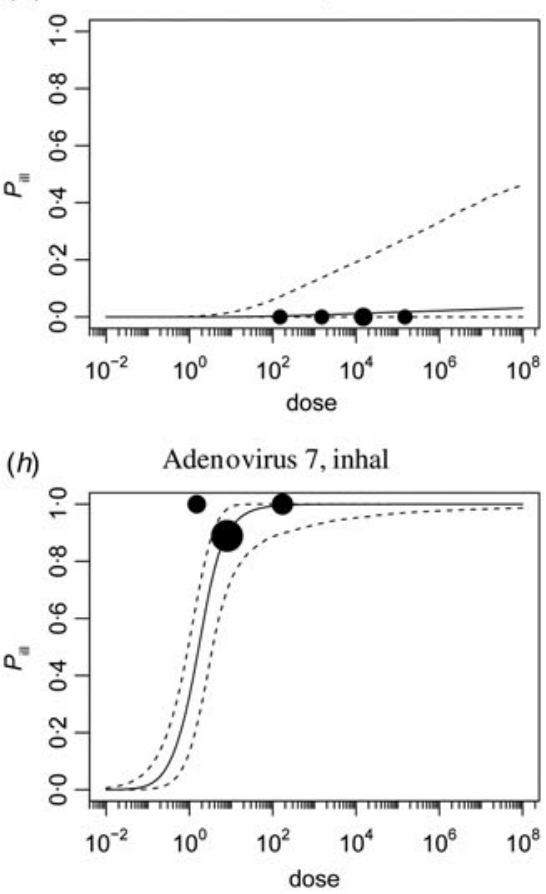

Fig. A3. Illness dose-response, separate studies. 

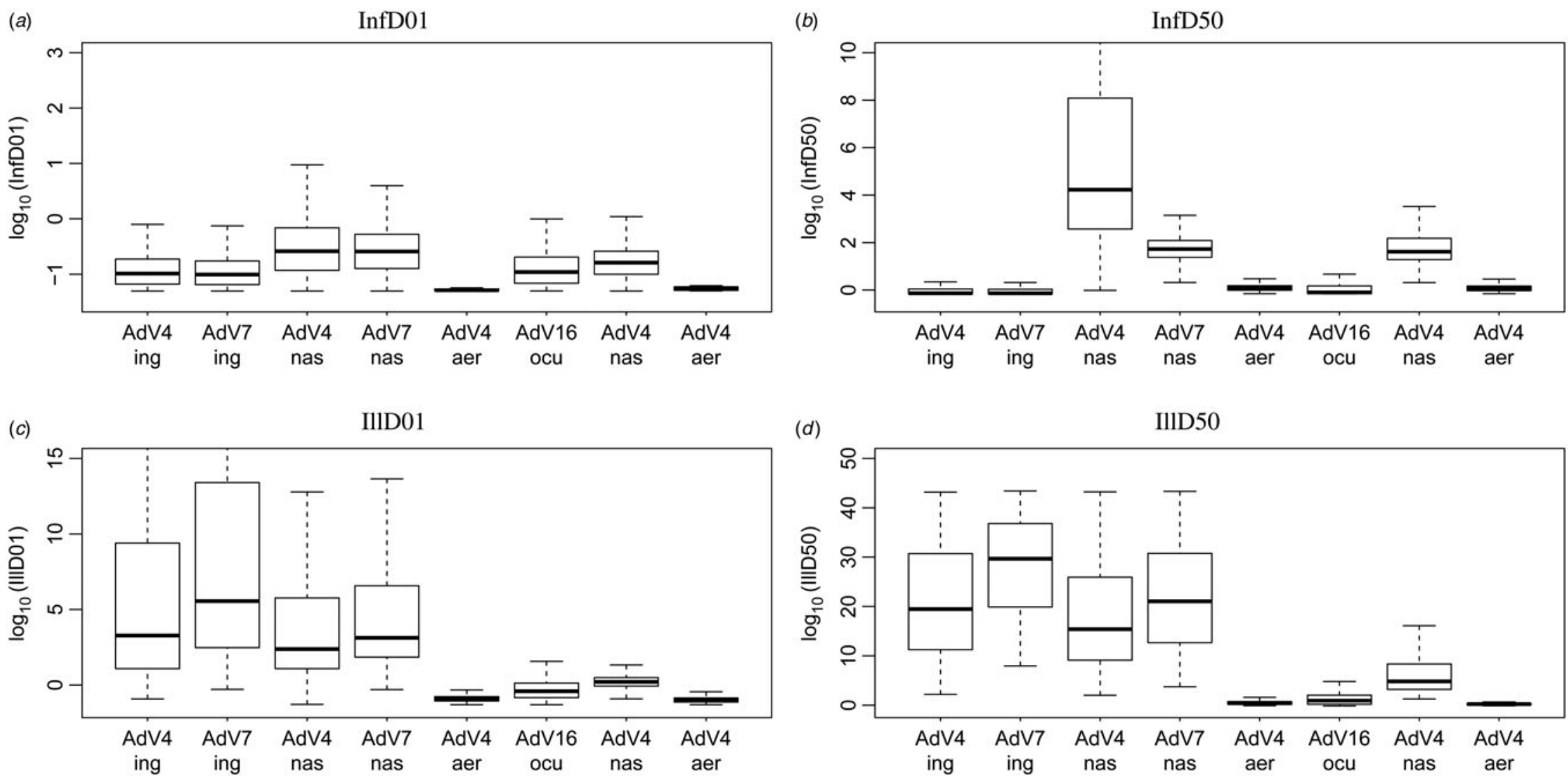

Fig. A4. Doses for $1 \%$ and $50 \%$ probability of infection and illness, for the separate studies. 


\section{Source code of the model is given below:}

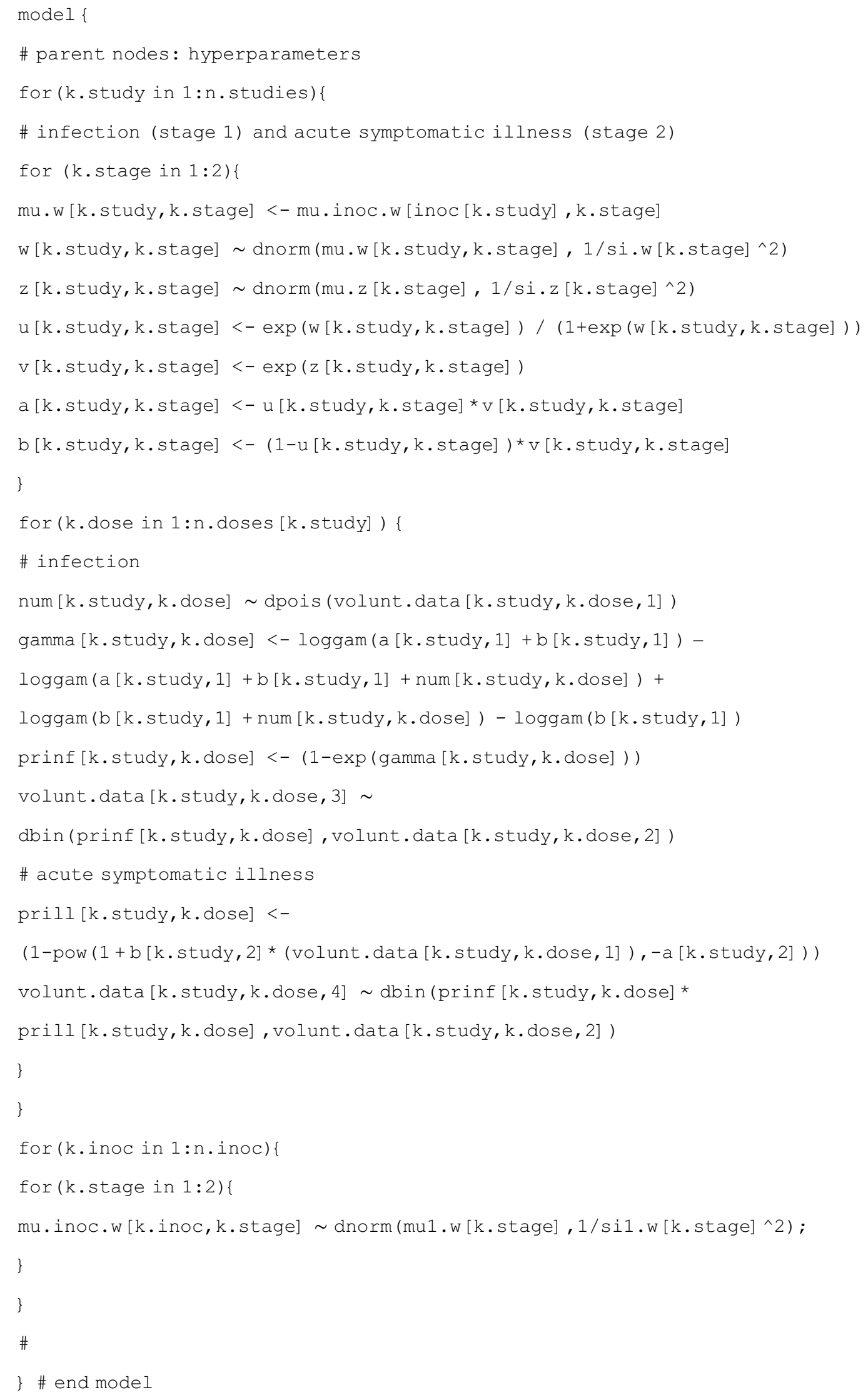




\section{ACKNOWLEDGEMENTS}

This paper received no fnancial support.

\section{DECLARATION OF INTEREST}

None.

\section{REFERENCES}

1. Ludwig SL, et al. Prevalence of antibodies to adenovirus serotypes 4 and 7 among unimmunized US Army trainees: results of a retrospective nationwide seroprevalence survey. Journal of Infectious Diseases 1998; 178: 1776-1778.

2. Garnett CT, et al. Prevalence and quantitation of species $\mathrm{C}$ adenovirus DNA in human mucosal lymphocytes. Journal of Virology 2002; 76: 10608-10616.

3. Gray GC, et al. Genotype prevalence and risk factors for severe clinical adenovirus infection, United States 20042006. Clinical Infectious Diseases 2007; 45: 1120-1131.

4. Brandt CD, et al. Infections in 18000 infants and children in a controlled study of respiratory tract disease. I. Adenovirus pathogenicity in relation to serologic type and illness syndrome. American Journal of Epidemiology 1969; 90: 484-500.

5. Bon F, et al. Prevalence of group a rotavirus, human calicivirus, astrovirus, and adenovirus type 40 and 41 infections among children with acute gastroenteritis in Dijon, France. Journal of Clinical Microbiology 1999; 37: 3055-3058.

6. Rodriguez-Baez N, et al. Astrovirus, adenovirus, and rotavirus in hospitalized children: prevalence and association with gastroenteritis. Journal of Pediatric Gastroenterology and Nutrition 2002; 35: 64-68.

7. Oh DY, Gaedicke G, Schreier E. Viral agents of acute gastroenteritis in German children: prevalence and molecular diversity. Journal of Medical Virology 2003; 71: 82-93.

8. Wishart PK, et al. Prevalence of acute conjunctivitis caused by chlamydia, adenovirus, and herpes simplex virus in an ophthalmic casualty department. British Journal of Ophthalmology 1984; 68:653-655.

9. Wold WSM, Ison MG. Adenoviruses. In: Knipe DM, Howley PM, eds. Fields Virology, 7th edn, chapter 56. Philadelphia: Lippincott, Williams \& Wilkins, 2013.

10. van Heerden $\mathbf{J}$, et al. Prevalence, quantification and typing of adenoviruses detected in river and treated drinking water in South Africa. Journal of Applied Microbiology 2004; 99: 234-242.

11. Choi S, Jiang SC. Real-time PCR quantification of human adenoviruses in urban rivers indicates genome prevalence but low infectivity. Applied and Environmental Microbiology 2005; 71: 7426-7433.

12. Bofill-Mas S, et al. Quantification of human adenoviruses in European recreational waters. Food and Environmental Virology 2010; 2: 101-109.

13. Wyn-Jones AP, et al. Surveillance of adenoviruses and noroviruses in European recreational waters. Water Research 2011; 45: 1025-1038.
14. Teunis PFM, et al. The dose response relation in human volunteers for gastro-intestinal pathogens. Technical Report 284550002, Rijksinstituut voor Volksgezondheid en Milieu RIVM, 1996.

15. Teunis PFM, Brienen N, Kretzschmar MEE. High infectivity and pathogenicity of influenza A virus via aerosol and droplet transmission. Epidemics 2010; 2: 215-222.

16. Kasel JA, Loda F, Knight V. Infection of volunteers with Adenovirus type 16. Proceedings of the Society for Experimental Biology and Medicine 1963; 114: 621-623.

17. Couch RB, et al. Immunization with types 4 and 7 adenovirus by selective infection of the intestinal tract. American Review of Respiratory Disease 1963; 88: 394403.

18. Couch RB, et al. Effect of route of inoculation on experimental respiratory viral disease in volunteers and evidence for airborne transmission. Bacteriological Reviews 1966; 30: 517-529.

19. Couch RB, et al. The minimal infectious dose of adenovirus type 4: the case for natural transmission by viral aerosol. Transactions of the American Clinical and Climatological Association 1969; 80: 205-211.

20. Hitchcock G, Tyrrell DAJ, Bynoe ML. Vaccination of man with attenuated live adenovirus. Journal of Hygiene (Cambridge) 1960; 58: 277-282.

21. Teunis PF, et al. Norwalk virus: how infectious is it? Journal of Medical Virology 2008; 80: 1468-1476.

22. Thebault A, et al. Infectivity of GI and GII noroviruses established from oyster related outbreaks. Epidemics 2013; 5: 98-110.

23. Teunis PFM, Havelaar AH. The Beta Poisson model is not a single-hit model. Risk Analysis 2000; 20: 511-518.

24. Teunis PFM, Nagelkerke NJD, Haas CN. Dose response models for infectious gastroenteritis. Risk Analysis 1999; 19: 1251-1260.

25. Teunis PFM, Ogden ID, Strachan NJC. Hierarchical dose response of $E$. coli O157:H7 from human outbreaks incorporating heterogeneity in exposure. Epidemiology and Infection 2008; 136: 761-770.

26. Teunis PFM, Takumi K, Shinagawa K. Dose response for infection by Escherichia coli O157:H7 from outbreak data. Risk Analysis 2004; 24: 401-408.

27. Plummer M. JAGS: a program for analysis of Bayesian graphical models using Gibbs sampling. In: Proceedings of the 3rd International Workshop on Distributed Statistical Computing (DSC 2003). Vienna, Austria, 2003, pp. 1-10.

28. Plummer M. rjags: Bayesian graphical models using MCMC, 2013 (http://CRAN.R-project.org/package= rjags). R package.

29. R Core Team. R: A Language and Environment for Statistical Computing. R Foundation for Statistical Computing, Vienna, Austria, 2015 (http://www. R-project.org/).

30. Strachan NJC, et al. Dose response modelling of Escherichia coli $\mathrm{O} 157$ incorporating data from foodborne and environmental outbreaks. International Journal of Food Microbiology 2005; 103: 35-47.

31. Ryan M, et al. Evaluating the potential for a Helicobacter pylori drinking water guideline. Risk Analysis 2014; 34: 1651-1662. 